\title{
Regionale Sicherstellung der Pflegeversorgung
}

Stefan Greß und Klaus Jacobs

Inhaltsverzeichnis

$13.1 \quad$ Einleitung -186

13.2 Status quo: Zuständigkeits- und

Verantwortungsdiffusion - 187

13.3 Sicherstellungsregelungen in der GKV:

Vorbild für die Pflegeversorgung? - 190

13.4 Leitplanken für die Gewährleistung

bedarfsgerechter Pflegeangebote - 192

Literatur - 193 


\section{- Zusammenfassung}

Heute sind die Länder verantwortlich für die Vorhaltung einer ,leistungsfähigen, zahlenmäßig ausreichenden und wirtschaftlichen pflegerischen Versorgungsstruktur", den Pflegekassen hingegen obliegt der individuelle Sicherstellungsauftrag, d. h., sie haben eine bedarfsgerechte Versorgung der Versicherten zu gewährleisten. Die Friktionen, die aus dieser Regelung resultieren, werden herausgearbeitet sowie potentielle Neuordnungen mit Blick auf die Verantwortlichkeiten diskutiert. Diese bestehen in der Entwicklung und verpflichtenden Anwendung einer Bedarfsplanung für die pflegerische Versorgung, in der gezielten Förderung von Versorgungsangeboten durch die Länder oder über die Versorgungsverträge der Pflegekassen und in einer deutlich verbindlicheren Kooperation der für Gewährleistung bedarfsgerechter Pflegeangebote zentralen Akteure.

Currently the German Länder are responsible for the provision of an "efficient, sufficient and economical nursing care structure", while the long-term care insurance funds are obliged to fulfil their individual guarantee mandate, i.e. they have to ensure that their insurees receive care that meets their needs. The authors describe the frictions resulting from this regulation and discuss potential reorganisations with regard to responsibilities. These are the development and obligatory application of needs planning for nursing care, the targeted promotion of care offers by the Länder or via the care contracts of the long-term care insurance funds and a much more binding cooperation of the central players for ensuring needs-oriented care offers.

\subsection{Einleitung}

Die Zahl der Pflegebedürftigen in Deutschland wird auch in den kommenden Jahren weiter wachsen - sowohl absolut als auch relativ als Anteil der Gesamtbevölkerung. Gleichzeitig wird es immer schwieriger, den hohen Anteil der in häuslicher Umgebung ambulant gepflegten Menschen auf dem heutigen hohen Niveau zu halten. Hierzu tragen neben demographischen Ursachen auch weitere gesellschaftliche Entwicklungen bei, wie etwa die gestiegene Frauenerwerbstätigkeit sowie die Zunahme der Mobilität, die dazu führt, dass Großeltern, Eltern und Kinder einer Familie immer seltener am selben Ort leben.

Auch wenn es speziell in der jüngeren Vergangenheit zahlreiche Anstrengungen gab, die Rahmenbedingungen für die „Pflege zu Hause" $z u$ verbessern, führt die skizzierte Entwicklung zwangsläufig zu einem immer höheren Bedarf an außerhäuslichen Pflegeangeboten. Das betrifft keineswegs allein das Pflegeheim als Ort der stationären Langzeitpflege, sondern auch viele intermediäre Pflegearrangements, die entweder einen zeitlich begrenzten Pflegeauftrag haben, wie speziell die Kurzzeitund die Verhinderungspflege, oder im Grenzbereich zwischen der eigenen Häuslichkeit und dem Pflegeheim angesiedelt sind.

$\mathrm{Da}$ es schon heute - zumindest in einzelnen Regionen - immer wieder Hinweise auf Engpässe bei den bestehenden Pflegeangeboten gibt, stellt sich die Frage, wie in Zukunft gewährleistet werden kann, dass der weiter wachsende Bedarf an außerhäuslichen Pflegeangeboten umfassend und möglichst bedarfsgerecht gesichert wird. Um mögliche Antworten auf diese Frage geht es in diesem Beitrag. Hierzu wird zunächst ein Blick auf den sozialrechtlichen Status quo im Sozialgesetzbuch Elftes Buch (SGB XI) geworfen, wo zwar eine Vielfalt an Verantwortlichkeiten definiert sind, im Ergebnis damit allerdings eher Verantwortungsdiffusion als eine klare Aufgabenzuordnung erzeugt wird. Deshalb wird im nächsten Schritt der Frage nachgegangen, ob sich die in der gesetzlichen Krankenversicherung (GKV) speziell in der vertragsärztlichen und der stationären Versorgung bestehenden Regelungen zu Bedarfsplanung, Zulassung und Sicherstellung auch im Pflegekontext als zweckmäßig erweisen könnten. Allerdings gibt es auch hier Reformbedarf, wie nicht nur 
an zahlreichen Versuchen der Weiterentwicklung der bestehenden sektoralen Regelungen abgelesen werden kann, sondern insbesondere auch an dem Bestreben, verstärkt sektorenübergreifende Ansätze zu verfolgen. Vor diesem Hintergrund wird im dritten Abschnitt der Versuch unternommen, zentrale „Leitplanken“ für die Gewährleistung eines bedarfsgerechten Angebots an außerhäuslichen Pflegeangeboten zu formulieren. Dies betrifft neben der Identifizierung von klaren Verantwortlichkeiten für einzelne Akteure - voran die Kommunen - nicht zuletzt auch die Frage von wirksamen Instrumenten, über die die Verantwortlichen verfügen müssen, um ihrer Sicherstellungsaufgabe hinreichend wirksam gerecht werden zu können.

\subsection{Status quo: Zuständigkeits- und Verantwortungsdiffusion}

Das SGB XI enthält in Bezug auf die $\mathrm{Zu}-$ ständigkeiten einzelner Akteure eine Vielzahl von Regelungen, die in einer Übersicht in - Tab. 13.1 dargestellt sind.

Es beginnt in $\S 8$ SGB XI mit der Feststellung, dass es sich bei der pflegerischen Versorgung der Bevölkerung um eine gesamtgesellschaftliche Aufgabe handelt, bei deren Wahrnehmung die Länder, die Kommunen, die Pflegeeinrichtungen und die Pflegekassen unter Mitwirkung des Medizinischen Dienstes eng zusammenwirken, um eine leistungsfähige, regional gegliederte, ortsnahe und aufeinander abgestimmte ambulante und stationäre pflegerische Versorgung zu gewährleisten, zum Ausbau und zur Weiterentwicklung der notwendigen pflegerischen Versorgungsstrukturen beizutragen und die Bereitschaft zu einer humanen Pflege durch hauptberufliche und ehrenamtliche Pflegekräfte sowie durch Angehörige, Nachbarn und Selbsthilfegruppen zu fördern. Wagner (in Hauck und Noftz 2019, $\mathrm{Rz} 5$ ) zufolge beruhe diese Vorschrift ,,auf der Erkenntnis, dass Pflegebedürftigkeit mit staatlichen (öffentlichen) Hilfesystemen allein nicht bewältigt werden“ könne, ,sondern nur im Zusammenwirken mit privaten (professionellen und ehrenamtlichen Hilfesystemen)“. Vorschriften mit Appellcharakter seien dem Sozialrecht nicht fremd, doch sei ihre Sinnhaftigkeit als fraglich anzusehen (Wagner in Hauck und Noftz 2019, Rz. 6).

Die nach $\S 8$ a SGB XI gebildeten Landespflegeausschüsse zur Beratung über Fragen der Pflegeversicherung sind nicht mehr als informelle Gremien; ,ihr letztlich unverbindlicher Charakter als ,runde Tische" zeigt sich auch darin, dass den Empfehlungen keine Bindungswirkung zukommt" (Reimer in Hauck und Noftz 2019, Rz. 6).

Eine zentrale Gestaltungsrolle bei der pflegerischen Versorgung weist das Gesetz den Ländern zu. Gemäß § 9 SGB XI sind sie verantwortlich für die Vorhaltung einer leistungsfähigen, zahlenmäßig ausreichenden und wirtschaftlichen pflegerischen Versorgungsstruktur. Wie das mit Hilfe von Planung und (Investitions-)Förderung geschehen soll, wird durch Landesrecht bestimmt, wobei es in Bezug auf die Planung große Unterschiede zwischen den Ländern gibt.

Die Investitionsförderung von Pflegeeinrichtungen durch die Länder ist seit Bestehen der Pflegeversicherung ein politisches Streitthema. Die damals eingeführte getrennte Finanzierungszuständigkeit - für die laufenden Betriebskosten durch die Pflegeversicherung, für die Investitionskosten durch die Länder - orientiert sich vom Grundsatz her am Modell der dualen Krankenhausfinanzierung. Im Pflegekontext ist jedoch meist von einer, ,unechten“dualen Finanzierung die Rede, weil für die Länder keine entsprechende Verpflichtung besteht und sie auch der gesetzlichen SollBestimmung, als Folge der Einführung der Pflegeversicherung bei der Sozialhilfe entstandene Einsparungen zur finanziellen Förderung der Investitionskosten der Pflegeeinrichtungen einzusetzen, praktisch von Anfang nicht oder bestenfalls in geringem Umfang gefolgt sind. Dabei spielt neben dem Haushaltsvorbehalt (Förderung nur nach Maßgabe der zur Verfügung stehenden Mittel) (Wagner in Hauck und Noftz 2019, Rz. 10) entgegen der erkennbaren 
- Tab. 13.1 Zuständigkeiten einzelner Akteure im Kontext der Sicherstellung der Pflegeversorgung im SGB XI

$\S 8$ (Gemeinsame Verantwortung)

§ 8a (Gemeinsame Empfehlungen zur pflegerischen Versorgung)

$\S 9$ (Aufgaben der Länder)

$\S 12$ (Aufgaben der Pflegekassen)

$\S 69$ (Sicherstellungsauftrag)

$\S 72$ (Zulassung zur Pflege durch Versorgungsvertrag)
Pflegerische Versorgung der Bevölkerung als gesamtgesellschaftliche Aufgabe; enges Zusammenwirken von Ländern, Kommunen, Pflegeeinrichtungen und Pflegekassen unter Beteiligung des Medizinischen Dienstes zur Gewährleistung einer leistungsfähigen, regional gegliederten, ortsnahen und aufeinander abgestimmten ambulanten und stationären pflegerischen Versorgung der Bevölkerung

Durch Rechtsverordnungen auf Landesebene gebildete Landespflegeausschüsse können einvernehmlich Empfehlungen zur Weiterentwicklung der Versorgung abgeben, die von den Vertragsparteien beim Abschluss von Versorgungs- und Vergütungsverträgen einbezogen werden sollen

Verantwortung der Länder für die Vorhaltung einer leistungsfähigen, zahlenmäßig ausreichenden und wirtschaftlichen pflegerischen Versorgungsstruktur; Bestimmungen zu Planung und Förderung der Pflegeeinrichtungen durch Landesrecht

Verantwortung der Pflegekassen für die Sicherstellung der pflegerischen Versorgung ihrer Versicherten; dabei enge Zusammenarbeit mit allen an der pflegerischen, gesundheitlichen und sozialen Versorgung Beteiligten und Hinwirken auf eine Vernetzung der regionalen und kommunalen Versorgungsstrukturen

Gewährleistung einer bedarfsgerechten und gleichmäßigen, dem allgemein anerkannten Stand medizinisch-pflegerischer Erkenntnisse entsprechenden pflegerischen Versorgung der Versicherten durch die Pflegekassen; hierzu Abschluss von Versorgungsverträgen sowie Vergütungsvereinbarungen mit den Trägern von Pflegeeinrichtungen und sonstigen Leistungserbringern

Zulassung von Pflegeeinrichtungen durch Versorgungsverträge, die Art, Inhalt und Umfang der Pflegeleistungen festlegen und zwischen dem Träger der Einrichtung und den Landesverbänden der Kassen im Einvernehmen mit den Sozialhilfeträgern geschlossen werden

Pflege-Report 2021

Intention der Gesetzesbestimmungen auch die Auffassung der Nichtzuständigkeit eine Rolle. So enthielt etwa ein im Bundesrat im Februar 2019 eingebrachter Entschließungsantrag der Länder Hamburg, Berlin, Bremen und Schleswig-Holstein zur Weiterentwicklung der Pflegeversicherung - u. a. mit Einführung eines dynamisierten Zuschusses aus dem Bundeshaushalt - in der Begründung die Aussage, es sei ,breit akzeptiert“, dass die Pflegebedürftigen im Pflegeheim die Kosten für Wohnen und allgemeinen Lebensunterhalt (Investitionskosten im Sinne von Kaltmiete und Vergütung für Unterkunft und Verpflegung) selbst trügen; dies stelle auch einen Aspekt der Gleichbehandlung von häuslicher und vollstationärer
Pflege dar (Bundesrat 2019, S. 3). Dies ist allerdings eine gewagte Position, denn zumindest bei der Einführung der Pflegeversicherung wurde für die stationäre Pflege von einer dreigeteilten Finanzierungsverantwortung ausgegangen. Danach sollte die Pflegeversicherung die pflegebedingten Kosten tragen, die (um Sozialhilfeausgaben entlasteten) Länder die Investitionskosten und die Pflegebedürftigen die Kosten von Unterkunft und Verpflegung (Rothgang et al. 2019, S. 14 f.).

Wenn die Länder der ihnen zugewiesenen Aufgabe der Investitionsförderung nur wenig oder gar nicht nachkommen, verzichten sie zugleich auf ein wesentliches Instrument der gezielten Einflussnahme auf das Ange- 
bot an Pflegeeinrichtungen. Dies gilt ebenfalls, wenn sie die ihnen seit 2008 eingeräumte Option nutzen, Pflegebedürftige beim Tragen von Investitionskosten zu unterstützen - also eine Objektförderung von Einrichtungen durch eine Subjektförderung von Pflegebedürftigen $\mathrm{zu}$ substituieren, wie es etwa in Nordrhein-Westfalen in Gestalt des Pflegewohngelds für bedürftige Pflegebedürftige geschieht.

Während den Ländern im Gesetz die Verantwortung für die pflegerische Versorgungsstruktur zugewiesen wird, sind die Pflegekassen nach $\S 12$ SGB XI für die Sicherstellung der pflegerischen Versorgung ihrer Versicherten zuständig. Konkretisiert wird diese allgemeine Aufgabenzuweisung in $\S 69$ SGB XI. Dort wird ihr Sicherstellungsauftrag dahingehend definiert, dass die Pflegekassen eine bedarfsgerechte und gleichmäßige, dem allgemein anerkannten Stand medizinisch-pflegerischer Erkenntnisse entsprechende pflegerische Versorgung der Versicherten zu gewährleisten haben. Hierzu schließen sie Versorgungsverträge sowie Vergütungsvereinbarungen mit den Trägern von Pflegeeinrichtungen und sonstigen Leistungserbringern. Dies bedeutet auch, dass die Pflegekassen Leistungen in der Regel nicht selbst erbringen, sondern dass diese Leistungen von selbstständigen Leistungserbringern erbracht werden, unter denen pflegebedürftige Versicherte eine Auswahl treffen können (Becker in Hauck und Noftz 2019, Rz. 25).

Der Abschluss von Versorgungsverträgen durch die Pflegekassen konstituiert zugleich die Zulassung der vertragschließenden Pflegeeinrichtungen gemäß $§ 72$ SGB XI. Sofern diese die in $\S \S 71$ und 72 Abs. 3 angeführten Anforderungen erfüllen, besteht für sie ein Anspruch auf Abschluss eines Versorgungsvertrags. Dieser Rechtsanspruch spiegelt den Willen des Gesetzgebers wider, ,die Pflege durch eine Vielfalt und ein Überangebot von Leistungserbringern sicherzustellen" (Groth in Hauck und Noftz 2019, Rz. 33).

Damit ergibt sich für den Auftrag der Sicherstellungsverantwortung der Pflegekassen folgende Situation: Für den Fall, dass das Pflegeangebot den (wie auch immer konkret festgestellten) ,geplanten Bedarf“ übersteigt (Überangebot), haben die Pflegekassen keine Möglichkeit auszuwählen. Zwar gibt es in $\$ 72$ Abs. 3 SGB XI eine Vorschrift, wonach Versorgungsverträge ,bei notwendiger Auswahl zwischen mehreren geeigneten Pflegeeinrichtungen $(. .$.$) vorrangig mit freigemeinnützi-$ gen und privaten Trägern abgeschlossen werden (sollen)", doch ist diese Regelung angesichts des bereits erwähnten Rechtsanspruchs der Pflegeeinrichtungen auf Zulassung, wegen der eine Auswahlentscheidung gar nicht notwendig werden kann, ,bestenfalls überflüssig“ (Groth in Hauck und Noftz 2019, Rz. 34). Zudem stünde sie im Widerspruch zur Intention des Gesetzgebers, durch ein Überangebot an Leistungserbringern Wettbewerb zu generieren (vgl. ebenda). Dabei handelt es sich allerdings erkennbar um ein Wettbewerbsverständnis, bei dem die Pflegekassen als Akteure nicht vorkommen - anders als etwa beim Vertragswettbewerb in der GKV, bei dem die Kassen bestimmte Versorgungsangebote selektiv unter Vertrag nehmen und ihre Versicherten eine entsprechende Tarifwahloption haben (Jacobs 2020).

Für den umgekehrten Fall, dass das Pflegeangebot den für erforderlich gehaltenen Bedarf nicht deckt (Unterangebot) - sei es im Hinblick auf den Gesamtbedarf an Pflegeplätzen vor Ort oder im Hinblick auf ganz bestimmte Angebote, etwa im Bereich der Kurzzeitpflege - müssen die Pflegekassen im Prinzip darauf vertrauen, dass die Länder ihrer Verantwortung für die Vorhaltung einer ausreichenden pflegerischen Versorgungsstruktur gerecht werden, damit überhaupt genügend Anbieter als potenzielle Vertragspartner bereitstehen, die die erforderlichen Voraussetzungen erfüllen. Erst dann können die Kassen ihre Aufgabe erfüllen, durch den Abschluss von Versorgungsverträgen ,dafür Sorge zu tragen, dass die für die Versorgung der Versicherten notwendige Zahl an Leistungserbringern zugelassen wird und dass diese Leistungserbringer ein solches Leistungsspektrum eröffnen, das den quantitativen 
Bedarf ihrer Versicherten erfüllt" (Becker in Hauck und Noftz 2019, Rz. 23).

Trotz vielfältiger Aufgabenzuweisungen an unterschiedliche Akteure und des gesetzlichen Appells zur Zusammenarbeit gibt es somit eine „Verantwortungslücke“, wenn es darum geht, mit einer - drohenden oder bereits akuten - Konstellation von Unterversorgung umzugehen, die ,der Markt“ nicht von selbst $\mathrm{zu}$ verhindern in der Lage ist. Im Grunde scheint die Konstellation eines Unterangebots im ,rechtlichen-faktischen Zuständigkeitsregime“"gar nicht vorgesehen zu sein. Sie könnte angesichts des wachsenden Bedarfs an professionellen Pflegeangeboten - speziell in eher dünn besiedelten Regionen, aber nicht nur dort - immer mehr Realität werden und bedarf deshalb einer frühzeitigen Antwort des Gesetzgebers bzw. der durch ihn mit der Sicherstellung eines bedarfsgerechten Pflegeangebots betrauten Akteure.

\subsection{Sicherstellungsregelungen in der GKV: Vorbild für die Pflegeversorgung?}

Die Pflegeversicherung ist im Vergleich zur Krankenversicherung ein vergleichsweise junger Zweig der Sozialversicherung. In der GKV gibt es daher bereits langjährige Erfahrungen mit Fragen der Bedarfs- und Kapazitätsplanung und Sicherstellung. Wir fassen in diesem Abschnitt daher diese Erfahrungen am Beispiel der ambulanten und stationären gesundheitlichen Versorgung zusammen. Darüber hinaus analysieren wir auch die Auswirkungen der sektorspezifischen Regelungen im Hinblick auf die sektorübergreifende Versorgung.

Die ambulante haus- und fachärztliche Versorgung in der GKV unterliegt im Hinblick auf die Bedarfsplanung und die Zulassung einer hohen Regulierungsdichte. Die Freiheit zur Niederlassung ist stark eingeschränkt, zumindest wenn die betroffenen Haus- und Fachärzte gesetzlich versicherte Personen behandeln und mit der GKV abrechnen wollen. Die reine privatärztliche Niederlassung ist nicht beschränkt - ist aber wegen des geringen Anteils von Privatpatienten in der Bevölkerung in der Regel betriebswirtschaftlich nicht attraktiv. Insofern werden in der GKV dezentrale Niederlassungsentscheidungen der Ärzte durch zentrale Planungsprozesse zumindest beeinflusst. $\mathrm{Zu}$ unterscheiden sind in diesem Zusammenhang die Bedarfsplanung und das den Akteuren zur Verfügung stehende Instrumentarium zur Sicherstellung einer bedarfsgerechten ambulanten ärztlichen Versorgung.

Die Bedarfsplanung in der ambulanten ärztlichen Versorgung erfolgt auf der Basis bundeseinheitlicher Regelungen. Diese werden im Grundsatz im SGB V normiert und - wichtiger noch - durch Regelungen des Gemeinsamen Bundesausschusses operationalisiert. Die aus der Bedarfsplanung abgeleiteten Verhältniszahlen (Arzt-Versicherten-Relationen) sind die Grundlage für die formalrechtliche Definition von Über- und Unterversorgung und die aus diesen Definitionen resultierenden Interventionsmöglichkeiten. Ursprünglich wurde diese Bedarfsplanung eingeführt, um gegen eine vom Gesetzgeber Anfang der 1990er Jahre befürchtete Überversorgung in der ambulanten ärztlichen Versorgung vorgehen zu können. Insbesondere im letzten Jahrzehnt hat sich die politische Zielsetzung der Bedarfsplanung jedoch verschoben. Im Mittelpunkt steht nun vielmehr die Beseitigung regionaler Disparitäten. Hierzu zählt nicht nur die Unterversorgung in vielen ländlichen Regionen, sondern auch die Gleichzeitigkeit von Über- und Unterversorgung in Ballungsräumen. In diesem Zusammenhang wurde die Bedarfsplanung mehrfach reformiert, um eine kleinräumigere, am tatsächlichen Bedarf orientierte Planung zu ermöglichen. Diese ist immer noch nicht perfekt. Insbesondere Ballungsräume bestehen vielfach immer noch aus einer Planungsregion, womit eine genauere Erfassung des Bedarfs innerhalb dieser Planungsgebiete verhindert wird. Nichtsdestoweniger sind deutliche Fortschritte in der Bedarfsplanung von einer am Status quo Anfang 
der 1990er Jahre orientierten Fortschreibung der Verhältniszahlen hin zu echten Bedarfsindikatoren zu erkennen (Gemeinsamer Bundesausschuss 2020).

Für die Sicherstellung der ambulanten ärztlichen Versorgung sind die regionalen Kassenärztlichen Vereinigungen (KVen) zuständig. Die gesetzlichen Krankenkassen bzw. deren Verbände haben in diesem Zusammenhang auf Landesebene eine untergeordnete Bedeutung. Grundlage des Sicherstellungsauftrags der KVen ist die Bedarfsplanung, mit der unterversorgte Planungsbezirke identifiziert werden. Umgekehrt ist allerdings auch Überversorgung $\mathrm{zu}$ vermeiden, weil ärztliche Leistungserbringer in überversorgten Gebieten in unterversorgten Gebieten fehlen. Die schon oben angesprochenen regionalen Disparitäten in der regionalen ambulanten ärztlichen Versorgung bestehen jedoch trotz der Fortschritte in der Bedarfsplanung weitgehend unverändert fort. Das liegt zum einen daran, dass die KVen die vom Gesetzgeber zur Verfügung gestellten Instrumente nicht hinreichend genutzt haben. So sollten KVen dafür sorgen, dass Zulassungen von in den Ruhestand gehenden Praxisinhabern in überversorgten Regionen aufgekauft und dann stillgelegt werden. Dies ist nur in Ausnahmefällen geschehen, sodass ein Abbau von Überversorgung nicht möglich war. Zum anderen haben die regionalen Disparitäten auch Ursachen, die außerhalb des direkten Einflussbereichs der KVen liegen. So ist die Niederlassung in sozioökonomisch starken Regionen bzw. Stadtvierteln attraktiver als in sozioökonomisch benachteiligten Regionen - was nicht zuletzt am hohen Anteil von deutlich besser vergüteten Privatpatienten liegt. Nichtsdestoweniger gibt es vor dem Hintergrund der wenig erfolgreichen Bemühungen zur Sicherstellung von Seiten der KVen zunehmend Forderungen, den Ländern und vor allem den Kommunen mehr Kompetenzen in diesem Zusammenhang zu geben (Greß und Stegmüller 2017).

In der stationären Versorgung erfolgt die Planung der Kapazitäten nicht auf der Basis einer Bedarfsplanung mit bundeseinheitlichen
Kriterien. Zuständig sind hier vielmehr die Bundesländer, die im Rahmen der jeweiligen Krankenhausplanung die Zulassung zur stationären Versorgung regeln. Es gibt analog zur ambulanten ärztlichen Versorgung auch ausschließlich privat abrechnende Krankenhäuser, die aber einen nur geringen Marktanteil haben. Die Verantwortung für die Bedarfsplanung und die Sicherstellung der stationären Versorgung ist nicht wie in der ambulanten Versorgung geteilt, sondern befindet sich in einer Hand. Zudem sind die Bundesländer für die Finanzierung der Investitionskosten zuständig, die Betriebskosten müssen von den Krankenversicherungen getragen werden. Insbesondere diese geteilte Finanzierungsverantwortung hat dazu beigetragen, dass kein Land in Europa mehr Krankenhausbetten pro Kopf der Bevölkerung hat als Deutschland. In vielen Bundesländern wurden Überkapazitäten in der stationären Versorgung aufgebaut, weil die Ausweitung von stationären Kapazitäten politisch deutlich attraktiver ist als deren Abbau. Die in den letzten Jahren häufiger auftretende Schließung von Krankenhäusern war dann auch vor allem in der mangelnden Investitionskostenfinanzierung der Bundesländer und durch Anreize des Systems der Fallpauschalen begründet als durch Planungsentscheidungen der Bundesländer. Die fortgeschriebenen Krankenhauspläne haben dann die Entwicklungen auf dem Markt für stationäre Leistungen lediglich nachvollzogen.

Ein weiterer Kritikpunkt an den Regelungen zur Bedarfsplanung und Zulassung in den beiden beschriebenen Sektoren besteht darin, dass es keinen funktionierenden Mechanismus zur Berücksichtigung der Kapazitäten im jeweils anderen Sektor gibt. So wäre eine sektorübergreifende Versorgung zur Planung einer bedarfsgerechten Versorgung besonders in ländlichen Regionen sinnvoll (Greß und Schnee 2017). Erste Ansätze gibt es dazu in Form von regionalen Gesundheits- bzw. Versorgungskonferenzen, die im Regelfall aber weder über ein eigenes Budget noch über eigene rechtliche Planungskompetenzen verfügen. Ähnliches gilt auch für das 
Gemeinsame Landesgremium mit Vertretern des Landes, der Kassenärztlichen Vereinigung, der Krankenkassen, der Landeskrankenhausgesellschaft sowie weiteren Beteiligten nach $\S 90 \mathrm{a}$ SGB V.

\subsection{Leitplanken für die Gewährleistung bedarfsgerechter Pflegeangebote}

Die bisherigen Ausführungen zeigen, dass eine systematische Zuweisung von Verantwortlichkeiten zur Gewährleistung eines bedarfsgerechten Versorgungsangebots in der pflegerischen Versorgung alles andere als trivial ist. Dies zeigen auch die langjährigen Erfahrungen mit der Angebotsplanung in der gesundheitlichen Versorgung. Nichtsdestoweniger lassen sich aus unserer Sicht drei zentrale Schlussfolgerungen ableiten. Diese bestehen erstens in der Entwicklung und verpflichtenden Anwendung einer Bedarfsplanung für die pflegerische Versorgung, zweitens in der gezielten Förderung von Versorgungsangeboten durch die Länder oder über die Versorgungsverträge der Pflegekassen und drittens in einer deutlich verbindlicheren Kooperation der für Gewährleistung bedarfsgerechter Pflegeangebote zentralen Akteure.

Eine zentrale Voraussetzung für eine bedarfsgerechte Planung der pflegerischen Versorgung besteht darin, dass die zuständigen Institutionen belastungsfähige Informationen über den in einer Region bestehenden Bedarf haben. Diese Informationen liegen derzeit im Hinblick auf die pflegerische Versorgung in den Regionen - gemeint sind hier vor allem die Kommunen - nur im Ausnahmefall vor. Eine solche Ausnahme könnte darin bestehen, dass die Knappheit etwa von Angeboten von Plätzen in der Kurzzeitpflege durch Interventionen direkt bei kommunalen Mandatsträgern angezeigt wird. Eine systematische Erhebung des Bedarfs einschließlich einer Gegenüberstellung mit dem vorhandenen
Angebot in statischer und dynamischer Perspektiver findet jedoch nicht flächendeckend statt. Ein erster Schritt für die Gewährleistung bedarfsgerechter Pflegeangebote müsste daher in einer systematischen Feststellung des Bedarfs in den Kommunen bestehen, dem dann das regionale Angebot gegenübergestellt werden kann. Diese regionale Bedarfsplanung auf der Ebene der Kommunen müsste von den Bundesländern verantwortet und finanziert werden. Voraussetzung wäre eine entsprechende Erweiterung und Konkretisierung der Verantwortlichkeiten der Bundesländer in $\S 9$ SGB XI.

Die Implementierung einer Bedarfsplanung ist zwar eine notwendige, aber keine hinreichende Bedingung für die Gewährleistung bedarfsgerechter Pflegeangebote. Notwendig ist weiterhin ein effektives Instrumentarium zur Beeinflussung der Angebotsstrukturen insbesondere zur Verhinderung bzw. zum Abbau von Unterversorgung. Ein wesentliches Instrument hierzu gibt es bereits, nämlich die gezielte Förderung der Investitionsfinanzierung, durch die die Bundesländer die Entwicklung von bedarfsgerechten Versorgungsangeboten unterstützen könnten. Dies ist auch bisher schon möglich, beruht aber auf Freiwilligkeit. Die bisherigen Regelungen in diesem Zusammenhang sind so unverbindlich formuliert, dass die Länder die Verantwortung für die Investitionskostenfinanzierung auf die Pflegebedürftigen abschieben können. Damit verzichten sie allerdings auf ein zentrales Instrument, um pflegerische Versorgungsstrukturen beeinflussen zu können. Eine Veränderung der gesetzlichen Regelungen hin zu mehr Verbindlichkeit bei der Investitionskostenfinanzierung würde allerdings die Zustimmung einer Mehrheit der Bundesländer erfordern. Weil auch die Erfahrungen mit der Finanzierung der Investitionskosten der Krankenhäuser durch die Länder alles andere als positiv sind, erscheinen allerdings Zweifel angebracht, ob die Länder tatsächlich bereit sind, ihrer häufig reklamierten Verantwortung für die Daseinsvorsorge der Bevölkerung auch Taten in Gestalt eines substanziellen Beitrags zur Investitionsförderung 
einer bedarfsgerechten Pflegeinfrastruktur folgen zu lassen.

Vor diesem Hintergrund könnte ein anderes Instrument zur Umsetzung der Ergebnisse der Bedarfsplanung zweckmäßig sein, nämlich die Zulassung von Pflegeeinrichtungen durch Versorgungsverträge mit den Pflegekassen. Wie gezeigt haben diese zurzeit keine Auswahloption, weil alle Einrichtungen, die die gesetzlichen Anforderungen erfüllen, einen Anspruch auf den Abschluss eines Versorgungsvertrags haben. Damit soll erreicht werden, dass die Pflegebedürftigen eine möglichst große Zahl an Angeboten vorfinden, aus denen sie im Bedarfsfall auswählen können. Diese Regelung ist erkennbar auf eine Konstellation ausgerichtet, in der es keine Angebotsengpässe gibt - ein echtes Sicherstellungsproblem also gar nicht besteht. Weil hiermit jedoch zumindest stellenweise - zunehmend gerechnet werden muss, könnte es sich anbieten, die Verpflichtung der Pflegekassen zum Abschluss von Versorgungsverträgen von der jeweils festgestellten Bedarfsnotwendigkeit abhängig zu machen. Das hieße zum Beispiel, dass in einer Region so lange keine neuen Pflegeeinrichtungen eines bestimmten Typs (etwa der vollstationären Pflege) zugelassen würden, wie es bei anderen Angeboten (zum Beispiel der ambulanten oder teilstationären Pflege) Defizite gegenüber der Bedarfsplanung gäbe. Für den Fall fortbestehender Angebotsprobleme könnte letztlich auch darüber nachgedacht werden, Pflegekassen die Möglichkeit einzuräumen, Eigeneinrichtungen zu gründen, um ihrem Sicherstellungsauftrag gegenüber den Versicherten gerecht zu werden.

Gemäß § 8 SGB XI ist die pflegerische Versorgung der Bevölkerung eine gesamtgesellschaftliche Aufgabe mit der Definition der daraus folgenden Anforderungen an die betroffenen Akteure. $\mathrm{Zu}$ diesen Akteuren gehören nicht nur die Länder, sondern neben den gerade bereits angesprochenen Pflegeeinrichtungen und Pflegekassen nicht zuletzt die Kommunen. Auf den eher appellativen Charakter der Rechtsvorschrift haben wir bereits hingewiesen. Gleichwohl erscheint zur Ge- währleistung einer bedarfsgerechten pflegerischen Versorgung insbesondere dringend geboten, die Kommunen stärker einzubeziehen (Brettschneider 2019). Ein Instrument hierfür könnten die nach $\S 8$ a SGB XI bereits bestehenden Landespflegeausschüsse sein. Voraussetzung wäre allerdings, dass der Gesetzgeber die Verbindlichkeit zur Berücksichtigung der Empfehlungen dieses Gremiums bei der Planung der pflegerischen Versorgung und der Umsetzung der Planungsergebnisse deutlich erhöht.

Die genannten Maßnahmen würden - den politischen Willen vorausgesetzt - die Rahmenbedingungen für eine verbindliche $\mathrm{Ge}$ währleistung bedarfsgerechter Pflegeangebote deutlich verbessern. Allerdings ist $\mathrm{zu}$ befürchten, dass der seit Einführung der Pflegeversicherung beschrittene Pfad fortgesetzt wird. Dieser besteht im Wesentlichen darin, dass das Angebot der Versorgungsstrukturen primär durch Marktkräfte determiniert wird (Greß 2018; Pfundstein und Bemsch 2020). Ein durchgehender politischer Steuerungswille ist insbesondere auf der Ebene der Bundesländer nicht zu erkennen. Ausnahmen gibt es nur, wenn Länder und Kommunen entsprechende politische und finanzielle Verantwortung übernehmen (vgl. dazu den Beitrag von Braeseke et al. in diesem Band). Aus Sicht der Pflegebedürftigen hängt die Wahrscheinlichkeit für die Gewährleistung eines bedarfsgerechten Versorgungsangebots damit zum einen von betriebswirtschaftlichen Rationalitäten der Anbieter ab. Zum anderen sind die Pflegebedürftigen vom politischen Willen, diese Versorgungsstrukturen aktiv zu beeinflussen, und der finanziellen Ausstattung von Ländern und Kommunen abhängig.

\section{Literatur}

Brettschneider A (2019) Die Rolle der Kommunen: Ziele, Handlungsfelder und Gestaltungsmöglichkeiten kommunaler Pflegepolitik. In: Jacobs K, Kuhlmey A, Greß S, Klauber J, Schwinger A (Hrsg) Pflege-Report 2019: Mehr Personal in der Langzeitpflege - aber woher? Springer, Berlin Heidelberg, S 219-239 
Bundesrat (2019) Entschließung des Bundesrates zur Weiterentwicklung der Pflegeversicherung. Antrag der Länder Hamburg, Berlin, Bremen, Schleswig-Holstein. Drucksache 106/19 vom 01.03.2019

Gemeinsamer Bundesausschuss (2020) Richtlinie des Gemeinsamen Bundesausschusses über die Bedarfsplanung sowie die Maßstäbe zur Feststellung von Überversorgung und Unterversorgung in der vertragsärztlichen Versorgung. https://www.g-ba.de/ downloads/62-492-2391/BPL-RL_2020-12-17_iK2021-02-18.pdf (Erstellt: 17. Dez. 2020). Zugegriffen: 5. März 2021

Greß S (2018) Qualitätssicherung in der Langzeitpflege durch Wahlentscheidungen der Betroffenen? Eine ökonomische Perspektive. In: Jacobs K, Kuhlmey A, Greß S, Klauber J, Schwinger A (Hrsg) Pflege-Report 2018: Qualität in der Pflege. Springer, Berlin Heidelberg, S 147-153

Greß S, Schnee M (2017) Wege zur integrierten und sektorenübergreifenden Versorgung. Gesundh Ges Wissenschaft (ggw) 17(3):7-15

Greß S, Stegmüller K (2017) Rahmenbedingungen für eine effektive Versorgungssteuerung auf kommunaler Ebene. In: Brandhorst A, Hildebrandt H, Luthe E-W (Hrsg) Kooperation und Integration - das un- vollendete Projekt des Gesundheitssystems. Springer, Wiesbaden, S 375-385

Hauck, Noftz (2019) Sozialgesetzbuch, SGB XI - Soziale Pflegeversicherung, Loseblattkommentar (Stand: 9/2019). Erich Schmidt, Berlin

Jacobs K (2020) Die Pflegeversicherung: eine vertragswettbewerbsfreie Zone. In: Jacobs K, Kuhlmey A, Greß S, Klauber J, Schwinger A (Hrsg) Pflege-Report 2020: Neuausrichtung von Versorgung und Finanzierung, S 123-133

Pfundstein T, Bemsch M (2020) Vom Markt und den Sorgen - sollen individuelle Pflegeleistungen kommunal gesteuert werden? In: Jacobs K, Kuhlmey A, Greß S, Klauber J, Schwinger A (Hrsg) Pflege-Report 2020: Neuausrichtung von Versorgung und Finanzierung, S 149-163

Rothgang H, Kalwitzki T, Cordes J (2019) Alternative Ausgestaltung der Pflegeversicherung II (AAPV II): bedarfsgerecht - ortsunabhängig bezahlbar. Gutachten im Auftrag der Initiative Pro-Pflegereform, Bremen. https://www.propflegereform.de/fileadmin/default/Gutachten/2. Gutachten_AAPV_-_Langfassung.pdf. Zugegriffen: 5. März 2021

Open Access Dieses Kapitel wird unter der Creative Commons Namensnennung 4.0 International Lizenz (http:// creativecommons.org/licenses/by/4.0/deed.de) veröffentlicht, welche die Nutzung, Vervielfältigung, Bearbeitung, Verbreitung und Wiedergabe in jeglichem Medium und Format erlaubt, sofern Sie den/die ursprünglichen Autor(en) und die Quelle ordnungsgemäß nennen, einen Link zur Creative Commons Lizenz beifügen und angeben, ob Änderungen vorgenommen wurden.

Die in diesem Kapitel enthaltenen Bilder und sonstiges Drittmaterial unterliegen ebenfalls der genannten Creative Commons Lizenz, sofern sich aus der Abbildungslegende nichts anderes ergibt. Sofern das betreffende Material nicht unter der genannten Creative Commons Lizenz steht und die betreffende Handlung nicht nach gesetzlichen Vorschriften erlaubt ist, ist für die oben aufgeführten Weiterverwendungen des Materials die Einwilligung des jeweiligen Rechteinhabers einzuholen. 